\title{
Modeling Verbal and Nonverbal Interaction \\ - a Catastrophe Theoretic Contribution to the Inter- grammar of Arndt and Janney
}

\begin{abstract}
The article deals with the interpretive framework of Arndt and Janney's Intergrammar. An intergrative modelisation of verbal, prosodic and kinesic choices in speech is proposed within the methodology of catastrophe theory in order to analyze the dynamics of the functional relationships between these unifying aspects of face-to-face communication.
\end{abstract}

\section{Introduction}

In their book Intergrammar. Toward an integrative model of Verbal, Prosodic and Kinesic Choices in Speech ${ }^{1}$, H. Arndt and R. W. Janney outline an interpretive framework to acccount for language, prosody, and kinesics as a trimodal patterning in face-to-face interaction. They call this theoretic and methodological approach to verbal and nonverbal communicative behavior: the Intergrammar framework.

Leaving out the cognitive and conative dimensions of speech, the study focuses on the emotive communication only as a subject of relevance for the Intergrammar. Emotive communication, i. e. cross-modal displays of transitory affective states during conversation, is analysed as an interrelated complex of verbal, vocal, and kinesic choices which the speaker must relate to his communicative intentions strategically in order to interact adequately with respect to his partner and the specific setting. The Intergrammar thus presents itself as a 'schema of hypotheses about how the conventions regulating multimodal speech choices can be plausibly explained' 2 . In this sense it is but preliminary to 'a unified, crossmodal, regulative grammar of speech, in which language, prosody, and

\footnotetext{
* Steffen Nordahl Lund

Odense University

Campusvej 55

5230 Odense $M(D K)$

1 (1987).

2 P. 328
} 
kinesics are integrated within a single system and approached from a single perspective' 3 .

The aim of this article is to modelise a possible unified system of such cross-modal communication. To do so I shall adopt an integrative perspective from a catastrophe theoretic point of view. However, I do not, by some false presumption, propose the regulative grammar searched for. My purpose is merely, on the one hand, to apply catastrophe theory in a concrete linguistic analysis and, on the other hand, to orientate the static schema of the Intergrammar towards an overt dynamic integrative modelisation; and, moreover, to do so in a hopefully enlightening systematic manner.

In the following we shall briefly look at catastrophe theory as a methodological approach to the study of dynamic systems and introduce - in slightly more detail - to one catastrophe model in particular: the so-called butterfly, as it is by means of this model that we shall transform Arndt and Janney's proposals into catastrophe theoretic terms.

\section{Methodological approach}

Although catastrophe theory was presented for the first time more than twenty years ago there has still - to my knowledge - been no application of it from an interactional point of view in the field of applied linguistics. This is not to say that catastrophe theory has not had considerable impact on linguistics. Indeed it has, e. g. the works of René Thom who himself saw the potential of the catastrophe theory for the study of language from the very beginning of his research ${ }^{4}$. But so far the theory has mostly been applied in the morphogenetic field of word semantics (Thom; Wildgen) or in the ontogenetic field of 'pure' (as opposed to 'empirical') linguistics (Petitot) ${ }^{5}$. Nevertheless a catastrophist methodological approach to formalizing applied linguistics seems highly appropriate since interaction and interactional cross-modality are obviously dynamic processes with regard to the recurrent transitions of attitudinal states, verbally, vocally, and kinesically. It seems promising, therefore, to interpret cross-modal communication in terms of a theory developped for dynamic, changing systems, i. e.: in terms of catastrophe theory6.

3 Ibid.

4 See for example Thom (1975) and (1980).

5 See for example Thom (1975, 1980); Wildgen (1981, 1982); and Petitot (1985, 1989, 1991).

6 As there exists a great many general introductions to catastrophe theory (see for example Arnold (1984); Poston and Stewert (1978); Saunders (1980); Woodcock andDavis 
The catastrophe theory was first synthesized by the French mathematician René Thom in his book from 1972: Stabilité structurelle et morphogénèse $e^{7}$ The book marks a shift in the paradigm of intelligibility (in the Kuhnian sense) due to a radically new mathematical method of analysing and describing phenomena related to as diverse domains as physics, biology, sociology, and linguistics. The creation of word meaning is as proper an object of catastrophic analysis as the breaking of a wave or the differentiation of a cell. In each case the same mathematical language and the same modeling are used for describing the development or change of form (morphogenesis) regardless of the substance of the morphology.

According to a major thesis of catastrophe theory, any dynamic system can be determined by studying its behaviour near critical points, named singularities, that is, in calculus, all the points (maxima, minima, and points of inflection) of a graphic curve where the slope of the graph is level (where the first derivative of the function describing the qualitative behaviour of the system is equal to zero). The dynamic is said to be governed by a potential function (figure 1). This means that it constantly seeks a position of stable equilibrium, depicted by local minima of the potential, the local maxima corresponding to positions of unstable equilibria, and the points of inflection to the discontinuous transitions from one stable state of equilibrium to another. These transitions, or catastrophic jumps, can themselves be located as structurally stable. Elementary catastrophe theory is the determination of such stable discontinuities ${ }^{8}$.

Fig. 1. Graph of potential function

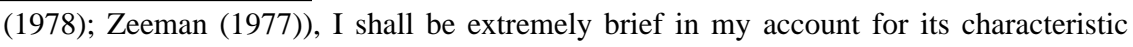
features.

7 English translation by D. H. Fowler (1975): Structural Stability and Morphogenesis. Reading, Mass.: Benjamin.

Thom's ideas had been circulating among scientists, though, since the mid-sixties.

8 By the suitable perturbations of the singularity, i. e. by addition of a minimum number of variable parameters in order to determine all possible stable states (the universal unfolding) of the potential. By application of Thom's classification theorem, the discontinuous catastrophe points will then appear as stabilised catastrophe subsets of the equilibrium surface. 
A given system can be specified by a finite, though potentially very large, number $n$ of internal or state variables $\left(x_{1}, x_{2}, \ldots x_{n}\right)$, determined by $m$ external or control variables $\left(a_{1}, a_{2}, \ldots a_{\mathrm{m}}\right)$. In fact this is called the 'splitting lemma' as it specifies the system by splitting the variables into two classes: the state variables describing the qualitative behaviour of the system in the internal space $\mathrm{R}^{\mathrm{n}}$ at a given point, and the control variables stabilising the former in the external space $\mathrm{R}^{\mathrm{m}}$. In this way the equilibrium surface of the system, partitioned into stationary points of minimum potential and catastrophe points of qualitative change, depends, not on the complex and often unmanageable or even undefinable internal state variables, but on the unfolding, external control variables 9 .

Now, the catastrophe theory says, in any structurally stable system (i. e. resistant to small quantitative perturbations) of one or two state variables, determined by no more than four control variables, only seven qualitatively different types of discontinuous behaviour can occur ${ }^{10}$. Thom calls these seven discontinuities the elementary catastrophes. Morphologically, the word 'catastrophe' is thus used in a broader and, generally, less dramatic sense than in everyday language. In the Thomian vocabulary, it designates a sudden discontinuous change in a smooth, continuously changing system.

The elementary catastrophes can be modelised by graphs depicting their geometric structure and topological features of both continuous and discontinuous change. We shall look a little further into one such model: the butterfly catastrophe.

\section{The butterfly}

The canonical form of the butterfly is given by the potential function:

$$
f(x)=x^{6}+a x^{4}+b x^{3}+c x^{2}+d x
$$

This catastrophe represents the stationary state stability of a system $x$ parametrized by four control variables $(a, b, c, d)$.

The equilibrium surface $M=\{(x, a, b, c, d) \mid \partial f / \partial x=0\}$ is given by the equation:

$$
f^{\prime}(x)=6 x^{5}+4 a x^{3}+3 b x^{2}+2 c x+d=0
$$

9 And what is more: not on their nature, only on their number. That is why catastrophe theory is applicable to such a wide variety of phenomena.

10 Other ways of change are of course conceivable, but either they are merely continouous variations, or they are unstable and thus, statistically, unlikely to happen (e. g. a falling pin landing on its head). 
and the catastrophe set $K=\left\{(a, b, c, d) \mid \partial f / \partial x=0\right.$ and $\left.\partial^{2} f / \partial x^{2}=0\right\}$ is the solutions of (1) and (2):

$$
f^{\prime \prime}(x)=30 x^{4}+12 a x^{2}+6 b x+2 c=0 .
$$

$\mathrm{K}$ is four-dimensional and therefore it cannot be drawn graphically. We shall have to do with series of two-dimensional cross sections in the ( $a$, $b$ )-plane for different values of $(c, d)$ :

Fig. 2. Sections of the butterfly catastrophe set. The effect of the factor $\mathrm{c}$ is to bias the position of the cusp and to move the surface up and down. The effect of the factor $d$ is to create the pocket (after Zeeman 1977).

The butterfly determines all possible continuous evolutions and discontinuous transitions of trimodal dynamics, e. g.:

Thus the most complex situation of the potential $\mathrm{f}(\mathrm{x})$ is at the triple point in the central area of the catastrophe ${ }^{11:}$

11 Only a few significant potentials are indicated. For a complete representation of the geometry of the butterfly catastrophe, see Petitot (1985a), 329-342. 
Fig. 3. Potentials at the triple point of the butterfly catastrophe.

In the following, we shall apply this trimodality of the butterfly as an integrative methodological model of the verbal, prosodic, and kinesic patterning in emotive communication.

\section{Modeling trimodal interaction}

According to Arndt and Janney, a major concern of speakers is to protect ones public self-image or face. In this way, two types of needs are distinguished as especially relevant to maintaining face: 1) the personal faceneed, defined as 'the need for personal autonomy (i. e. independence, self-determination, an inviolable private sphere)'; and 2) the interpersonal face-need, defined as 'the need for interpersonal acceptance (i. e. approval, respect, appreciation).' Conflicts arise if one partner feels either of these needs ignored or threatened in face-to-face interaction (1987, 378).

Let $\mathrm{a}=$ personal face-need and $\mathrm{b}=$ interpersonal face-need be two conflicting control factors: 'From a psychological point of view, autonomy and acceptance are in certain aspects antithetical. Autonomy is often realized only at the cost of lower acceptance, and acceptance is often purchased only at the cost of lower autonomy. As a result, personal and interpersonal face-needs often conflict...' (Arndt and Janney 1987, 378).

A third factor of relevance to interactional emotive communication is what Arndt and Janney call emotional security. The effect of the emotional security factor is to modulate speaker's communicative behaviour as either threatening or saving his partner's face. This means that the values of emotional security bias the possible intersections of the conflicting 
factors so as to predispose their combinatory values: 'High levels of emotional security produce increases in liking and intimacy and decreases in reciprocity and information seeking; low levels of emotional security produce decreases in liking and intimacy and increases in reciprocity and information seeking (Arndt and Janney 1987, 380). In other words: high emotional security values correspond to positive bias and faceneeds are likely to be met; low emotional security values correspond to negative bias and face-needs are threatened.

Any communicative situation is a potential face threat and needs to be constantly ratified (verbally, prosodically, and kinesically) if a speaker wishes to avoid conflicts and convey interpersonal intentions. A fourth control factor of emotive communication might therefore be what Arndt and Janney refer to as supportiveness: 'Supportiveness reduces emotional uncertainty, one of the causes of aggressiveness and anxiety, or fightflight reactions (Arndt and Janney 1987, 380). In our model, c = emotional security and $\mathrm{d}=$ supportiveness are called the bias factor and the butterfly factor respectively.

Whereas the effect of the bias factor is to outline the area of conflicting personal and interpersonal face-needs and thus to predetermine their intersecting values, the effect of the butterfly factor is, at positive values, to create a 'pocket of compromise' as a third modality of stationary minimum potentials between the two stable equilibrium surfaces of conflict points (cf. figure 2). The modulating strategies are here referred to as 'compromising' or 'supportive strategies'.

This leads us to the question of scaling the model. How do we account for the external variables? How do we parametrize their control?

If supportive speech strategies determine the supportiveness factor, we need to define, on the one hand, what might be called the management of these strategies and, on the other hand, the measurement of their dynamics.

Arndt and Janney (1987) observe: 'A supportive speaker smooths over uncomfortable situations, or keeps delicate situations from becoming interpersonally threatening, by acknowledging his partner's claim to a positive public self-image. He does this by verbally, prosodically, and kinesically confirming his partner's intrinsic worth as a person.' Supportive strategies then rely on 'impression management' as a technique to 'interact smoothly' by reducing potential face threats: 'A supportive speaker avoids undercutting his partner's personal and interpersonal 
face-needs. He indicates whenever possible that he does not wish to restrict his partner's freedom of action, and he shows that he respects his partner's feelings and does not wish to insult him by being unduly critical, aggressive or contemptuous.' In short: 'A supportive speaker tries to minimize territorial transgressions and maximize signs of interpersonal acceptance' (379-380).

Such signs, ascribed to emotive verbal, prosodic, and kinesic cues, thus constitute the measurement of the dynamics of supportive (and subsequently, nonsupportive) strategies. According to the naturally reductive schemata elaborated by Arndt and Janney they relate, in the verbal mode, to formal/informal style; direct/indirect strategy; in the prosodic mode, to high/low pitch prominence; falling/rising/falling-rising pitch; and in the kinesic mode, to smile/frown; full/averted gaze; tense/relaxed body (cf. 1987, figure 72).

Supportive strategies, while seeking a compromising state of the emotive dimensions of speech ${ }^{12}$, deal dynamically with the patterning relationship between verbal, prosodic, and kinesic cues and their emotive functions as governed by the external control variables. We shall therefore have to look at these variables and the complex interrelationship between them.

In this unified perspective, the bias factor, as related to the positivenegative dimension of speech, is the scaling of the valueladenness managed by displays of positive and negative affect cues in the three modes: '...valueladenness has to do with positively and negatively-biased ways of alluding to topics' (Arndt and Janney 1987, 346). Measurement is primarily value-laden language and facial expressions; secondarily, tone of voice, inclusiveness and exclusiveness of reference, explicitness and inexplicitness of reference, body posture, cross-modality and redundancy of verbal and non-verbal cues (cf. 345-351).

The face-need controls are related to the confidence and the involvement dimensions of speech scaling cues of assertiveness and involvement managed by expressions of self-confidence or uncertainty, respecti-

12 I. e. the confidence, the positive-negative, and the involvement dimensions: 'Three emotive dimensions of speech have been isolated and given considerable attention in clinical and social psychology: (1) the confidence dimension, or control dimension [ ], which is inferred from cues of assertiveness/nonassertiveness or self-confidence/insecurity; (2) the positive-negative dimension, or evaluative dimension, which is inferred from cues of positive/negative valueladenness; and (3) the involvement dimension, or intensity dimension, which is inferred from cues of emotional or interpersonal involvement/uninvolvement' (Arndt and Janney (1987), 330). 
vely expressions of interpersonal concern in the three modes. Measurement, with regard to the personal face need control, is primarily cues of verbal directness and indirectness and pitch direction; secondarily, gaze and gaze aversion, verbal formality and informality, variations in pitch prominence, frowning and smiling, body relaxation, and speaker-and partner-oriented utterances (cf. 338-345).

Measurement of the interpersonal face-need control is primarily cues of verbal formality/informality, and gaze/gaze aversion ${ }^{13}$; secondarily, impersonal, partner-oriented, and inclusive utterances, and smiling (cf. 357-360) ${ }^{14}$.

Arndt and Janney present the essential features in a schematic overview of a trimodal framework of emotive cues (figure 4).

From a catastrophe theoretic point of view, we can represent these features - and their implications for face-work - by means of the topology of the butterfly. In order to do so we shall apply examples of cross-modal patterning to effective strategies of emotive communication.

Before analysing, however, the topologisation of face-to-face interaction, we shall briefly outline two types of cross-modal patterning relevant to our purpose, namely redundant patterning and constrastive patterning: 'Redundant patterning occurs whenever primary verbal emotive cues are confirmed by corresponding primary cues in the prosodic and/or kinesic modes' (367); 'Contrastive patterning [ ] occurs whenever verbal behaviour is not sufficiently confirmed by redundant nonverbal behaviour, or when it is openly contradicted by nonverbal behaviour' (369).

Whereas redundancy across modes supports and amplifies verbal messages, disambiguating any eventual vagueness of the communicative intention, contrastive cross-modal cues modulate and modify verbal messages, deceiving a clear unambiguous notice of the communicative be-

13 Cf.: 'Levels of speech formality define relatively broad parameters of potential involvement for the partners in a given conversation. Gaze and gaze aversion, on the other hand, make finer distinctions within these parameters in a moment-to-moment basis' (Arndt and Janney (1987), 358).

14 We leave out of our model the emotional or personal involvement cues ('more or less spontaneous expressions of momentary personal affective states' (Arndt and Janney (1987), 351)) as part of the involvement dimension related to the interpersonal face-need control. These cues (primarily: verbal intensity, pitch prominence, and body posture; secondarily: sudden increases in informality, sudden increases in directness, falling pitch in utterances normally requiring a rise, rising pitch in utterances normally requiring a fall, gaze and gaze aversion (cf. 352-357)) seem likely to be interpreted as related to the confidence dimension of the personal face-need control as signs of assertiveness/nonassertiveness or self-confidence/insecurity. 
150

Figure 4 
haviour. Moreover: 'Studies of cross-modal communication show that when verbal and nonverbal behaviour is discrepant, people tend to interpret the former in terms of the latter, using nonverbal behaviour as a sort of check on the validity, dependability or sincerity of the verbal message' (369).

\section{Strategies for modulating interpersonal assertiveness}

Maximally threatening partner's face-needs in conveying interpersonal intentions is the redundant cross-modal patterning of nonsupportive selfassertive strategies, e. g. ${ }^{15}$ (the pitch direction`symbolizes falling intonation):

\section{(1) I want it to be put over 'THERE (frowning) (tense) (full gaze)}

Primary assertiveness cues of verbal directness and nonverbal cue redundancy of falling pitch, extreme pitch prominence, and body tension imply strong personal involvement and low interpersonal acceptance. We might therefore represent the dynamic of this utterance as a minimum of potential in parameter space at high values of personal face-need and low values of interpersonal face-need, and at negatively biasing values of affect cues (effect of facial expressions) (see figure 5).

Somewhat less aggressive, because emotionally or personally uninvolved, is the contrastive cross-modal patterning of nonsupportive nonassertive strategies, e.g.:

(2) There is probably room for that over in the corner (unsmiling) (averted gaze)

Primary nonassertiveness cues of verbal indirectness and the modal expression ("probably") contrast with nonverbal displays of interpersonal uninvolvement (discrepant facial expressions: speaker neither smiles nor seeks eye contact with listener), implying emotional detachment on behalf of the speaker with respect to his partner's needs for autonomy and acceptance. The dynamic of the utterance can be represented as a graph of potential at neutral values of personal and interpersonal faceneeds, though still, as an effect of the cross-modal discrepancy, at negatively biasing values of affect cues.

Things change radically (i. e. qualitatively, or catastrophically) when they become positively involving by means of supportive self-assertive strategies, e. g.:

15 The following examples are from Arndt and Janney (1987), $381 \mathrm{ff}$. 
(3) Tom, put it over 'there, please (smiling) (full gaze)

Here, primary nonverbal cues of interpersonal involvement (speaker smiles and looks at his partner) contrast with primary verbal cues of assertive directness in order to compensate speaker's threat to his partner's face, thus creating what we shal call a 'compromise modality': 'The partner's potential loss of personal face is compensated for by an increase in interpersonal face' (382) (i. e. 'fronting' reference to the partner; positive formulaic adjunct). The utterance can be figured as a graph of three conflicting minima at equal values of interpersonal and personal face-needs, and at positive values of supportiveness.

Maximally acknowledging partner's face-needs is the redundancy of supportive nonassertive strategies, e. g. (the pitch direction ' symbolizes falling-rising intonation; the dotted line under there symbolizes unusually low pitch prominence):

(4) Tom, would you mind putting it over ' there, please (smiling) (full gaze)

Primary verbal and nonverbal nonassertiveness cues (indirectness, falling rising pitch, and secondary nonverbal cues of low pitch prominence, smile and eye contact) together with positively stressed cues of interpersonal involvement ('fronting' person reference; positive formulaic adjunct) give maximal support to partner's face-needs. The utterance can be represented as a minimum potential in parameter space at low values of personal face-need and high values of interpersonal face-need and, as a (catastrophic) effect of the redundancy across modes, at maximally increasing values of affect cues.

We illustrate the features of these patternings by a trajectory in the catastrophe set of the butterfly: 


\section{Strategies for modulating positive-negative affect}

Another emotive dimension of speech is the positive-negative affect. We obtain very much the same trajectory when graphically representing strategies for modulating positive and negative feeling.

Maximal threat to partner's face is produced by the so-called nonsupportive negative utterance, e. g.:

(5) 'Susan, that's absolutely 'RIDICULOUS (frowning) (tense) (full gaze)

Redundancy of primary assertiveness, negative affect, and interpersonal involvement cues in the three modes (i. e.: directness, falling intonation, full gaze; verbal intensity, extreme pitch prominence, body tension; negatively value-laden language, and, secondarily, early partner reference) exhibit maximum negative emotion. We might represent the dynamic of this utterance as a graph of potential at high values of personal faceneeds and low values of interpersonal face-needs, and at negatively biasing values of affect cues (cf. figure 5, 1).

Nonsupportive positive utterances, on the other hand, are characterized by cross-modal discrepancy of positive verbal cues (positively valueladen language) contrasting with non-positive cues in other modes (e. g.: negative, inexplicit references; nonassertive indirectness, falling-rising intonation, low pitch prominence; interpersonally uninvolving averted gaze and unsmiling facial expression):

(6) That's " one way of seeing it, I suppose (unsmiling) (averted gaze)

Positive verbal behaviour must be accompanied by positive nonverbal behaviour as well, in order not to be interpreted negatively. As stated earlier, verbal positiveness tends to be undercut by cross-modal contrasts. We might therefore represent the dynamic of utterance (6) as a minimum potential at mutually neutralizing values of personal and interpersonal face-needs, and, though verbally positive, still at negatively biasing values of affect cues (cf. figure 5,2).

A speaker might wish to compensate for criticizing his partner and does so by minimizing threats to partner's face and maximizing signs of interpersonal acceptance, e. g.:

(7) I wish I could ' agree with you, Susan (smiling) (full gaze)

Primary cues of nonassertiveness (indirectness, falling-rising intonation) contrast with primary cues of positiveness (positive value-laden language) and involvement (smile, full gaze and, secundarily, inclusive part- 
ner reference) in order to signal interpersonal supportiveness. We figure this compensating 'compromise modality' by a graph of potential with three conflicting minima at equal values of personal and interpersonal face-needs, and at positive values of supportiveness (cf. figure 5, 3).

Unqualified positiveness is achieved by the cross-modal redundancy of the supportive positive utterance, e. g.:

(8) 'Susan, that's a TERRIFIC idea (smiling broadly) (full gaze)

Primary verbal and nonverbal cues of assertiveness, positive affect, and interpersonal involvement (that is: directness, falling intonation, emphasis, and verbal and prosodic intensity; positively value-laden language, broad smile and, secondarily, explicit reference; full gaze and, secondarily, 'fronting' partner reference) provide maximum support for partner's face. We can represent the dynamics of utterance (8) as a graph of potential at low values of personal face-need and high values of interpersonal face-need, and at extreme, increasing values of positive bias (cf. figure 5, 4).

\section{Strategies for modulating interpersonal involvement}

The last emotive dimension of speech: the involvement dimension, also presents a series of behavioral strategies, catastrophically similar to the trajectories we have already depicted.

Thus the nonsupportive uninvolvement strategies focuses almost exclusively on the verbal mode, that is with no visible sign of emotion, e. g.:

(9) Good 'evening (unsmiling) (normal gaze)

Primary interpersonal uninvolvement cues of verbal formality deceive any emotive information necessary to satisfy partner's need for interpersonal face. We graphically depict utterance $(9)$ by the figure $(5,1)$.

Nonsupportive involvement utterances are characterized by positive involvement cues of verbal informality contrasting with the absence of positive involvement cues in the other modes (no reference to the partner, no smile, neutral gaze), e. g.:

(10) You said you weren't 'coming (unsmiling) (normal gaze)

We recognize the essential features of the dynamics in figure $(5,2)$.

As to the supportive uninvolvement strategies, utterances are positive but verbally formal. However, this verbal formality is compensated for in 
other modes (smiling, gazing; eventually emphatic pitch prominence and falling intonation, and 'fronting' partner reference), e. g.:

(11) Mrs. 'Jones, what a pleasant 'surprise (smiling broadly) (full gaze)

Recognizing partner's face-needs, the contrastive patterning of such compromising utterances can be illustrated by the graph of potential with three conflicting minima of figure $(5,3)$.

Maximum face for the partner is provided by the redundancy of the supportive involvement strategies. Utterances of this type are characterized by primary assertiveness, positive affect, and interpersonal involvement cues in all modes, e. g.:

(12) 'Jane, what a nice 'SURPRISE (smiling broadly) (full gaze)

Verbal directness, positiveness, and informality, secondarily 'fronting' partner reference, are prosodically articulated with extreme pitch nucleus prominence and given a falling intonation. The effect of supportive involvement is kinesically accentuated by broad smile and intense gaze at the partner. We represent these features by the graph of figure $(5,4)$.

\section{Conclusion}

Emotive communication is a dynamic process involving a constantly ongoing, multimodal interaction of verbal and nonverbal strategies for expressing and interpreting emotional signals of assertiveness, positiveness, and involvement, and consequently the Intergrammar of Arndt and Janney, as an integrative modeling of verbal, prosodic, and kinesic choices in speech, focuses on the functional relationships between these unifying pragmatic aspects of face-to-face communication. The analytical outcome of the trimodal framework is a condensated, systematic overview of the complex and subtle patterning of the emotive dimensions in speech.

Complementary to the (static) schemata of the patterning relationship between verbal and nonverbal behavior ('One of the strengths of the InterGrammar, we believe in fact, lies in its straightforward illustration of these functional relationships' (397)), an analysis of the interactional setting in terms of catastrophe theory seems to be able to illustrate, not only a (static) schemata of the relationships between behavioral data but the very dynamics of their communicative interplay. Major problems, though, remain to be solved. 
I do not argue for the relevancy and characteristics of the parametrisation in modeling the integrative framework (and subsequently the butterfly). Arndt and Janney already do so, and this paper is but a proposal of a catastrophic "transcription" of their findings. Still we have to point out the problems related to scaling and measuring data accurately. Catastrophe theory models in linguistic areas are not at all excluded from experimental testing and verification. The difficulties of precise scaling and measurement techniques, however, may reduce the models to no more than descriptive, suggestive, or speculative mathematical metaphors. Somewhat refined procedures are necessitated to improve this state of the art. Despite the lack of sophisticated methods of scaling and measurement, catastrophe theory modeling, as a mathematical coherent methodology, represents a promising contribution to the communicative studies of verbal and nonverbal interaction by accomodating a strongly integrated view of the many interconnected facets of speech in their dynamic observational-operational organization. Virtual, elaborated catastrophe theory models are thus, contrary to the schemata of the Intergrammar, to be viewed as concrete analyses of actual behavioral events. This also opens for neatly illustrative contrastive studies of multimodal patterns and their implications for face-work cross-culturally: 'An important upshot of this research would be the identification of areas of potential emotional misunderstanding between speakers from different cultures' (398). The topology of the catastrophe modeling seems highly appropriated for a depiction of such areas and consequently may help 'explaining intercultural conflict avoidance strategies' (398).

The catastrophe theory constitutes a consistent conceptual and methodological contribution to exploring face-to-face communication. It thus fully responds to the invitation that closes the Intergrammar of Arndt and Janney: 'Finally, verbal and nonverbal behavior forms a unified whole; we can only invite our colleagues to follow Pike's [ ] suggestion more than thirty years ago, and start working on theories and methodologies that treat it as such' (399).

\section{References}

Arnold, V. I. (1984): Catastrophe Theory. Berlin: Springer-Verlag.

Arndt , H. and Janney, R. W. (1987): InterGrammar. Toward an integrative Model of Verbal, Prosodic and Kinesic Choices in Speech. Berlin: Mouton Gruyter.

Petitot, J. (1985a): Les catastrophes de la parole. Paris: Maloine.

Petitot, J. (1985b): Morphogenèse du Sens I. Paris: PUF. 
Petitot, J. (1989): On linguistic import of catastrophe theory. In: Semiotica 74 (3/4), 179209. (English translation of Sur la signification de la théorie des catastrophes (1982). In Mathématiques et Sciences humaines 79, 37-74.)

Petitot, J. (1991): Syntaxe topologique et grammaire cognitive. In: Langage 103, 97-127.

Poston, T. and I. N. Stewert (1978): Catastrophe Theory and its Applications. London: Pitman.

Saunders, P. T. (1980): An Introduction to catastrophe theory. Cambridge: Cambridge University Press.

Thom, R. (1975): Structural Stability and Morphogenesis. Reading, Mass.: Benjamin (English translation of Stabilité structurelle et morphogénèse. W. A. Benjamin, Inc. Deuxième Édition, revue, corrigée et augmentée, Paris: Interédition 1977.)

Thom, R. (1980): Modèles mathématiques de la morphogenèse. Paris: Christian Bourgois.

Wildgen, W. (1981): Archetypal Dynamics in Word Semantics: An Application of Catastrophe Theory. In: Words, Worlds, and Contexts. New Approaches in Word Semantics, Hans-Jürgen Eikmeyer and Hannes Rieser (eds.), 234-296. Berlin, New York: Walter de Gruyter.

Wildgen, W. (1982): Catastrophe Theoretic Semantics. Amsterdam: Benjamin.

Woodcock, A. E. R. and M. Davis (1978): Catastrophe theory. New York: E. P. Dutton.

Zeeman, E. C. (1977): Catastrophe Theory. Selected Papers, 1972-1977. Massachusetts: Addison-Wesley. 
